CORPUS $\begin{aligned} & \text { Corpus } \\ & 12 \mid 2013 \\ & \text { Dialectologie : corpus, atlas, analyses }\end{aligned}$

\title{
Concordances géolinguistiques et anthroponymiques en Bretagne
}

Daniel Le Bris

\section{(2) OpenEdition \\ 1 Journals}

Electronic version

URL: http://journals.openedition.org/corpus/2339

DOI: $10.4000 /$ corpus.2339

ISSN: 1765-3126

Publisher

Bases; corpus et langage - UMR 6039

\section{Printed version}

Date of publication: 1 January 2013

Number of pages: 85-104

ISSN: 1638-9808

\section{Electronic reference}

Daniel Le Bris, "Concordances géolinguistiques et anthroponymiques en Bretagne », Corpus [Online], 12 | 2013, Online since 13 May 2014, connection on 08 September 2020. URL : http:// journals.openedition.org/corpus/2339; DOI : https://doi.org/10.4000/corpus.2339 


\title{
Concordances géolinguistiques et anthroponymiques en Bretagne ${ }^{1}$
}

\author{
Daniel LE BRIS \\ CRBC Brest, UBO-UEB
}

\section{Les sources}

Cette étude est fondée sur la comparaison des données anthroponymiques et géolinguistiques de la Basse-Bretagne, la zone celtique de la péninsule armoricaine. Il ne s'agit pas ici de mettre au point une méthode pour déterminer le berceau d'une famille à partir d'un nom de famille (NF). Seule une recherche historique poussée et établie à partir d'informations généalogiques permettrait d'obtenir des résultats, sans garantie toutefois de pouvoir remonter à la source du NF.

Notre analyse essaie d'abord de montrer dans quelle mesure la représentation cartographique de la répartition des patronymes permet de compléter l'examen des variations morpholexicales et phonétiques d'une aire linguistique déterminée. Les cartes anthroponymiques sont constituées en utilisant les Fichiers des noms patronymiques de 1891 à 1990 - France entière de l'INSEE : Institut National de la Statistique et des Etudes Economiques (INSEE 1999). Ces fichiers contiennent pour chaque département de France métropolitaine les noms de famille de toutes les personnes nées de 1891 à 1990 . Tout patronyme est donc répertorié par département en fonction de l'année de naissance et de la commune de naissance. Nos observations se limitent pour le moment aux cinq départements de la Bretagne historique à savoir : le Finistère, les Côtes-d'Armor, le Morbihan, l'Ille-et-Vilaine et la Loire-Atlantique.

1 Je tiens à remercier chaleureusement Jean Le Dû pour ses encouragements et ses observations à mener cette recherche en partie inspirée par ses études sur les anthroponymes de Bretagne (Le Dû 1987, 1988 \& 1989).

Corpus $n^{\circ} 12$ « Dialectologie : corpus, atlas, analyses » (2013), 


\section{LE BRIS}

Les fichiers patronymiques de l'INSEE regroupent les naissances selon quatre périodes de vingt-cinq ans chacune: 1891-1915, 1915-1940, 1941-1965 et 1965-1990. Seules les deux premières périodes s'échelonnant de 1891 à 1940 sont retenues. En effet, après la Seconde Guerre Mondiale, un nouveau système de protection sociale est mis en place en France : la Sécurité Sociale. Grâce à elle, les enfants vont progressivement tous naître dans les maternités au lieu d'être mis au monde au domicile de la mère ou des parents. Les naissances sont désormais répertoriées dans la commune de l'hôpital ou de la clinique la plus proche et ne sont plus comptabilisées dans la commune de la résidence parentale. C'est la raison pour laquelle nous avons seulement pris en compte les deux périodes antérieures à 1941 afin d'être quasi-certains que les naissances soient bien enregistrées dans la commune d'origine des parents.

Une telle base de données permet de cartographier tous les patronymes sur l'ensemble des cinq départements. Ce travail a été possible grâce à l'aide très précieuse de Michel Floch ${ }^{2}$, ingénieur informaticien, qui a développé un logiciel permettant de dresser des cartes à partir des fichiers patronymiques de l'INSEE. Le logiciel est toujours en cours d'élaboration puisque nous y intégrons de nouvelles données. Ainsi, dans le cadre de cet article nous utilisons également les naissances répertoriées par le Centre Généalogique du Finistère ${ }^{3}$. Cela permet de remonter dans certains cas jusqu'aux $\mathrm{XVI}^{\mathrm{e}}-\mathrm{XVII}^{\mathrm{e}}$ siècles et d'apporter davantage de densité historique aux visualisations cartographiques de ce département. Nous espérons pouvoir bientôt intégrer les fichiers patronymiques des autres départements bretons et intégrer ainsi l'enregistrement des naissances sur trois ou quatre siècles.

2 Ma plus vive reconnaissance et mes plus sincères félicitations à Michel Floch dont le talent et les très nombreuses heures de travail et de patience ont permis de mettre au point ce formidable logiciel cartographique sans lequel cette recherche demeurait impossible.

3 Mes plus prompts remerciements à Jean-François Pellan, Président du Centre Généalogique du Finistère et à Robert Marhic, informaticien de cette association, pour leur adhésion à notre projet et leur soutien sans faille. 
La cartographie des patronymes dessine des territoires parfois inattendus. Afin de tenter de les décrire et de les identifier nous les avons comparés aux cartes des atlas linguistiques suivants : l'Atlas Linguistique de Basse-Bretagne (ALBB) (Le Roux 1924-63), le Nouvel Atlas Linguistique de Basse-Bretagne (NALBB) (Le Dû 2001) et l'Atlas Linguistique de la Faune Marine de Bretagne (ALFMB) (Le Berre 2008).

\section{La géographie linguistique de la Basse-Bretagne}

Une première limite linguistique, marquée par une ligne de points noirs sur la carte 1, s'étire du nord au sud depuis l'ouest de Saint-Brieuc jusqu'à l'est de Vannes. Elle sépare à l'ouest l'aire celtique, la Basse-Bretagne, de l'aire gallo-romane, la Haute-Bretagne, située à l'est.

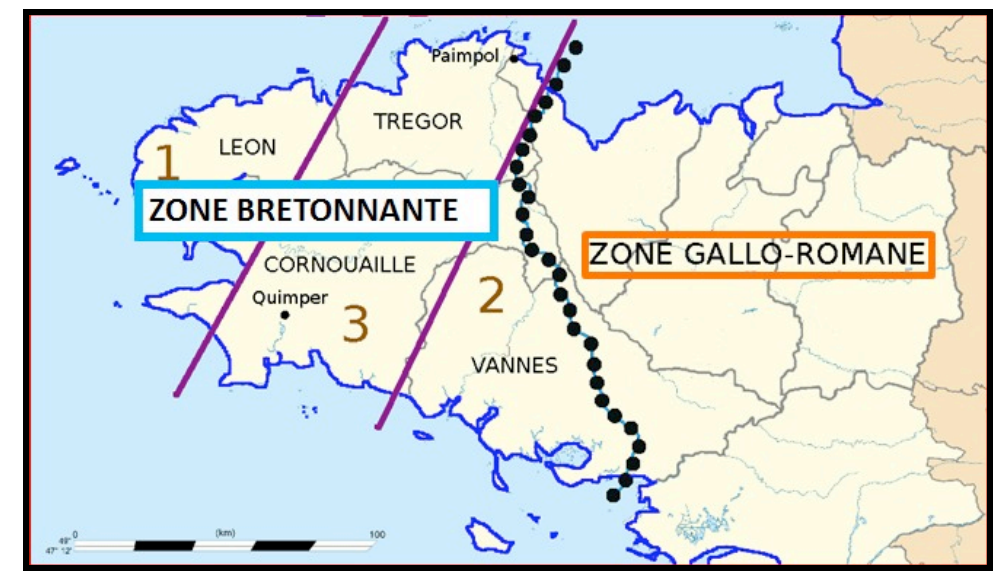

Carte 1. Dualité celtico-romane en Bretagne et zones dialectales de Basse-Bretagne

L'aire celtique ou zone bretonnante est caractérisée par une dualité dialectale entre les parlers du nord-ouest, qui correspondent aux territoires des anciens évêchés pré-révolutionnaires du Léon, Trégor et Cornouaille, et ceux du sud-est dont l'aire linguistique coïncide globalement avec l'ancien évêché de Vannes. Cette dualité s'établit selon une diagonale sud-ouest / nord-est qui s'étire depuis Quimper jusqu'à Paimpol. Plusieurs linguistes (Falc'hun 1981, Humphrey 1995, Plourin 1982), considèrent que cette transversale constitue elle-même une troisième 


\section{LE BRIS}

zone de dialectes et évoquent une tri-partition de l'espace linguistique breton.

Falc'hun et Fleuriot, jusqu'à un certain point, voient dans la dualité dialectale nord-ouest / sud-est l'illustration de l'arrivée, durant la fin de l'Antiquité et le haut Moyen-Age, des immigrants bretons de Grande-Bretagne qui auraient débarqués par groupes successifs sur la côte ouest-septentrionale de la péninsule armoricaine.

Faisons également remarquer que la ligne de cette bipartition dialectale (carte 2) concorde avec la limite qui séparait les territoires de deux tribus gauloises : les Ossismii au nordouest et les Veneti au sud-est (Merlat 1959, Fleuriot 1988, German 2007). Si cela était le cas, les variations dialectales s'inscriraient dans des schémas très anciens et les lisières lin guistiques mises en évidence remonteraient alors à l'Age des Métaux. La partie jaune sur la carte signale où se trouve la frontière ossismo-vénète selon Fleuriot (plus à l'ouest) ou selon Merlat (plus au sud-est).

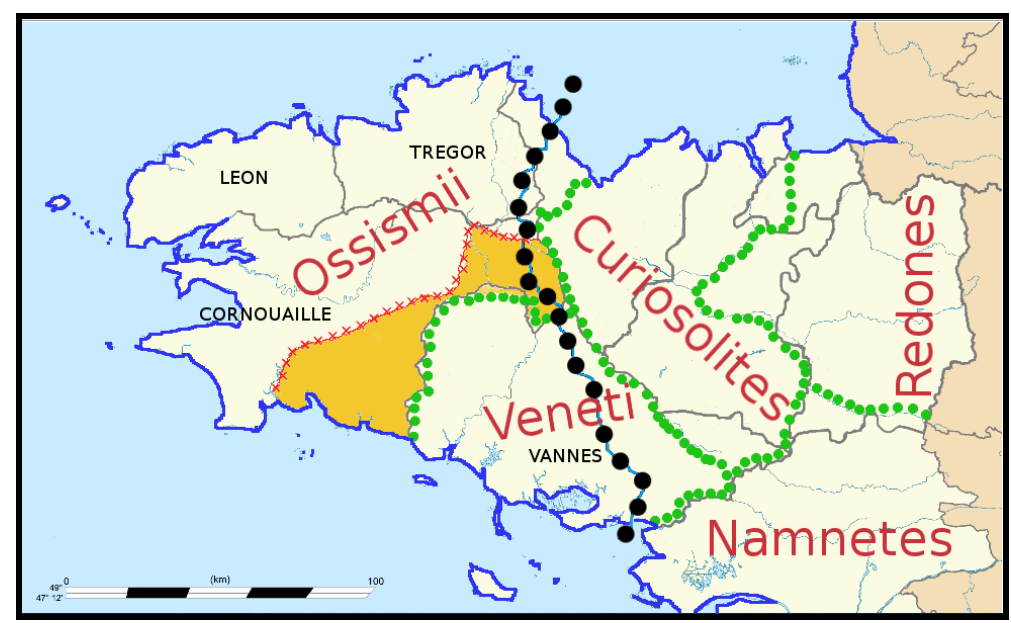

Carte 2. Anciens territoires gaulois en Armorique

\section{Dualité dialectale nord-ouest / sud-est}

La carte 3 représente l'ensemble des naissances enregistrées de 1891 à 1940 sous les patronymes Riou et Rio. Tous les deux sont des formes hypocoristiques du vieux-breton $r i$ « roi ». En 
rouge sur la carte, les naissances déclarées sous le NF Riou et en bleu, celles du NF Rio.

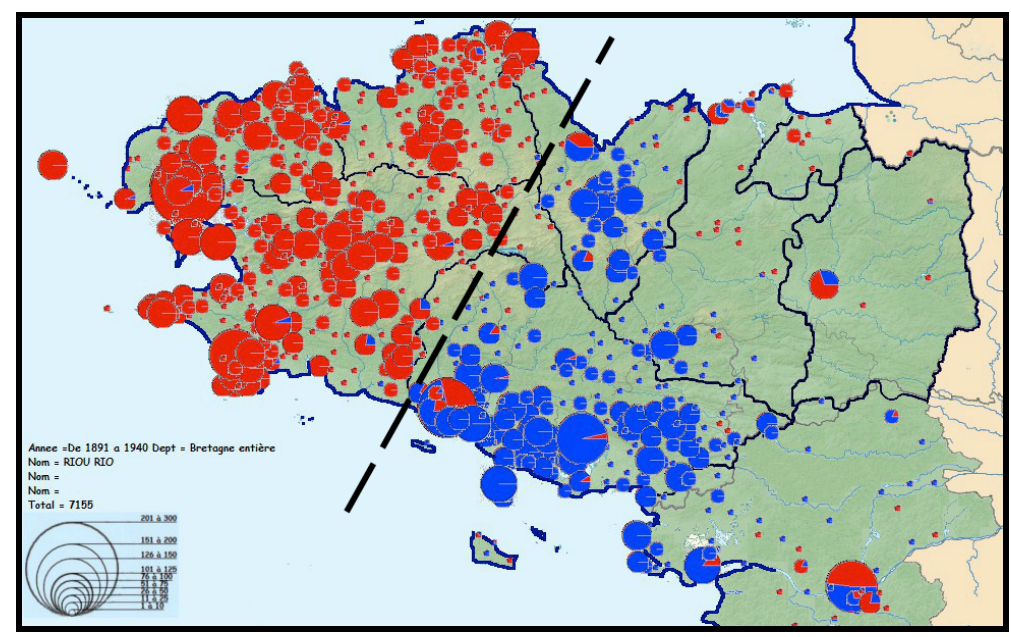

Carte 3. Patronymes Riou / Rio

Manifestement, l'aire de Riou correspond au tracé des anciens évêchés de Léon, Trégor et Cornouaille. Elle cadre avec la zone dialectale du nord-ouest et vient épouser au nord la limite linguistique celtico-romane. Au sud-est sa démarcation est quasi contigüe avec celle de l'ancien évêché de Vannes. Le patronyme Rio, variante sud-est du NF Riou, est quant à lui circonscrit au territoire de l'ancien évêché vannetais.

Le débordement du NF Rio à l'est de la frontière linguistique rappelle la zone d'extension des noms de lieu en Ker-. La densité de ce NL en Haute-Bretagne correspondrait selon Tanguy à la limite orientale des parlers celtiques vers les XII ${ }^{\mathrm{e}}-$ XIII $^{\mathrm{e}}$ siècles (Tanguy 1986).

Observons également que les deux NF sont en distribution complémentaire sur le territoire. Cette interdépendance de Riou et Rio est à rapprocher de la dualité dialectale de l'espace linguistique breton.

La carte 4 Jézéquel / Gicquel (en orange : Jézéquel, en bleu : Gicquel) visualise l'emplacement des variantes occidentale et orientale de l'anthroponyme vieux-breton Iudicael dont la plus ancienne attestation date du $\mathrm{IX}^{\mathrm{e}}-\mathrm{X}^{\mathrm{e}}$ siècle (Jackson 1967). 


\section{LE BRIS}

Remarquons que la localisation de ces deux variantes concorde une nouvelle fois avec la dualité dialectale nord-ouest / sud-est de Basse-Bretagne. Selon Jackson $(1953,1967)$, le « d » intervocalique reflétait un [ð]. La fricative interdentale sonore évolue ensuite en fricative alvéolaire dans le NF Jézéquel du nordouest et elle devient muette dans le sud-est. Nous constatons une nouvelle fois que la répartition sur le territoire de ces deux variantes correspond à la dualité dialectale nord-ouest / sud-est.

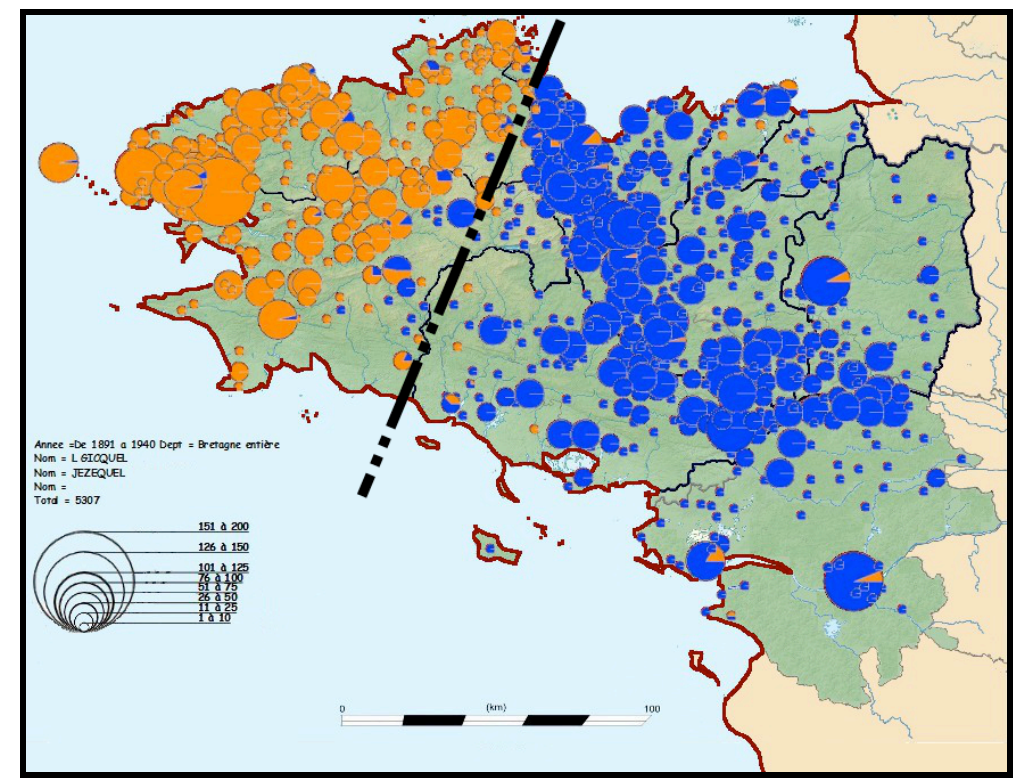

Carte 4. Patronymes Jézéquel / Gicquel

Le « $\mathrm{d}$ » intervocalique de IUDIC-HAEL reflétait peut-être déjà une fricative interdentale sonore qui évolue en fricative alvéolaire sonore dans la forme Jézéquel du nord-ouest et devient muette dans le sud-est.

La lisière est de la zone Gicquel nous rappelle à nouveau la limite orientale aux $\mathrm{XII}^{\mathrm{e}}-\mathrm{XIII}{ }^{\mathrm{e}}$ siècles des parlers celtiques.

\section{Evolution de la spirante dentale $[\Theta]$ du vieux-breton}

L'évolution de la spirante dentale $[\Theta]$ du vieux-breton est l'un des critères qui illustre le mieux la bipartition dialectale nord- 
ouest / sud-est en Basse-Bretagne. La fricative interdentale sourde devient $[\mathrm{z}]$ dans les parlers du nord-ouest et $[\mathrm{h}]$ dans le sud-est.

La carte 5 Mazé / Mahé met en lumière cette bipartition. En jaune, le NF Mazé est la variante nord-ouest du nom de l'apôtre Mattheus. Dans le sud-est, l'intervocalique de Mahé, en bleu, équivaut à une fricative glottale. L'existence de ces deux variations suppose une intervocalique ayant valeur de fricative interdentale au moment où Mattheus fut emprunté. La limite de bipartition dialectale est toujours évidente. Mais on la retrouve cette fois plus à l'ouest de la diagonale Quimper-Paimpol.

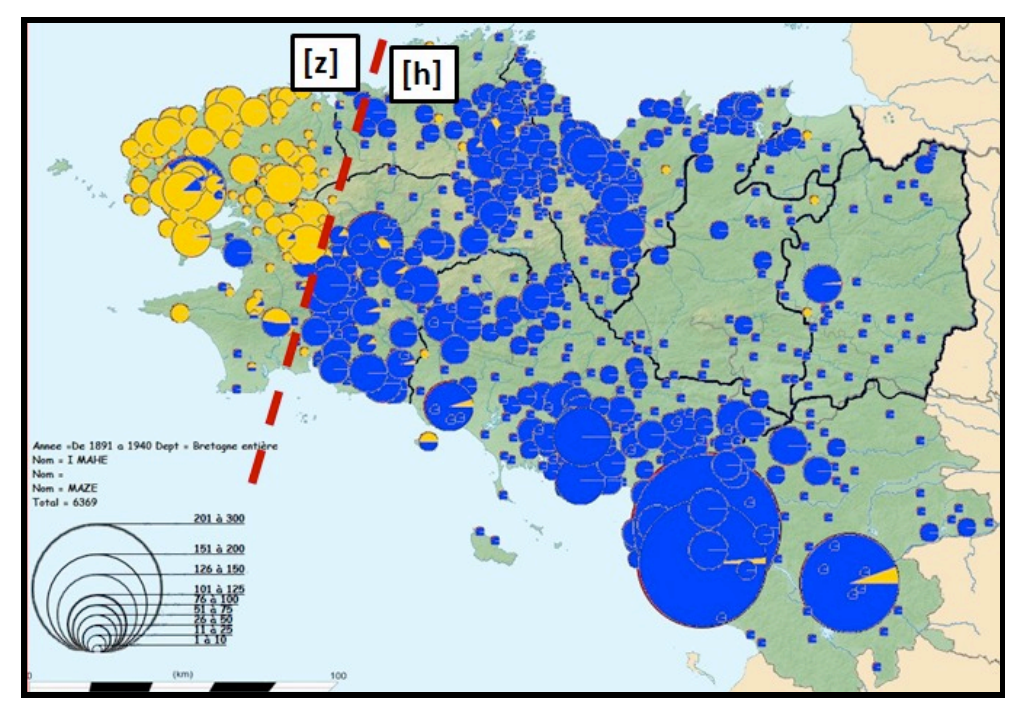

Carte 5. Patronymes Mazé / Mahé de 1891 à 1940

Les informations fournies par la carte 6 permettent de reconsidérer la représentation cartographique des NF Mazé/ Mahé sur la période 1701-1793. Il est frappant de constater que cette répartition est sensiblement la même à environ deux siècles d'intervalle. 


\section{LE BRIS}

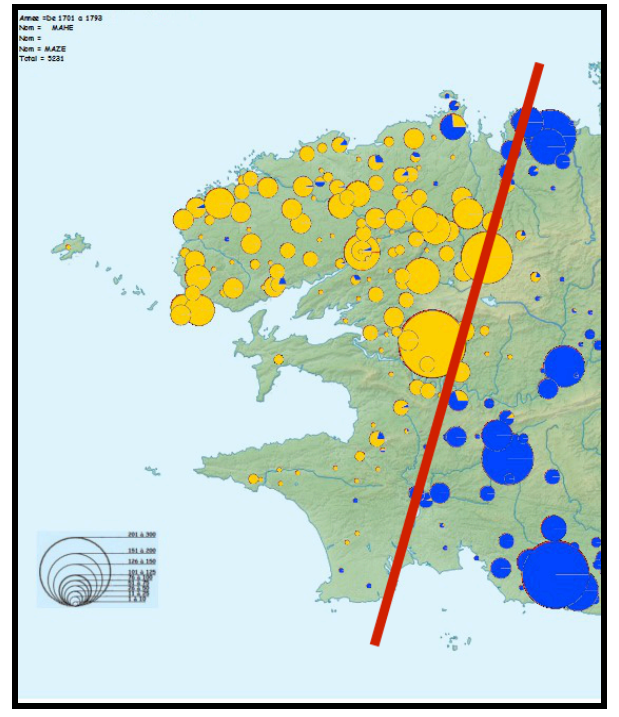

Carte 6. NF Mazé / Mahé 1701-1793

Sur la carte 7, nous avons reporté les données des NF continuateurs du nom vieux-breton Guethenoc.

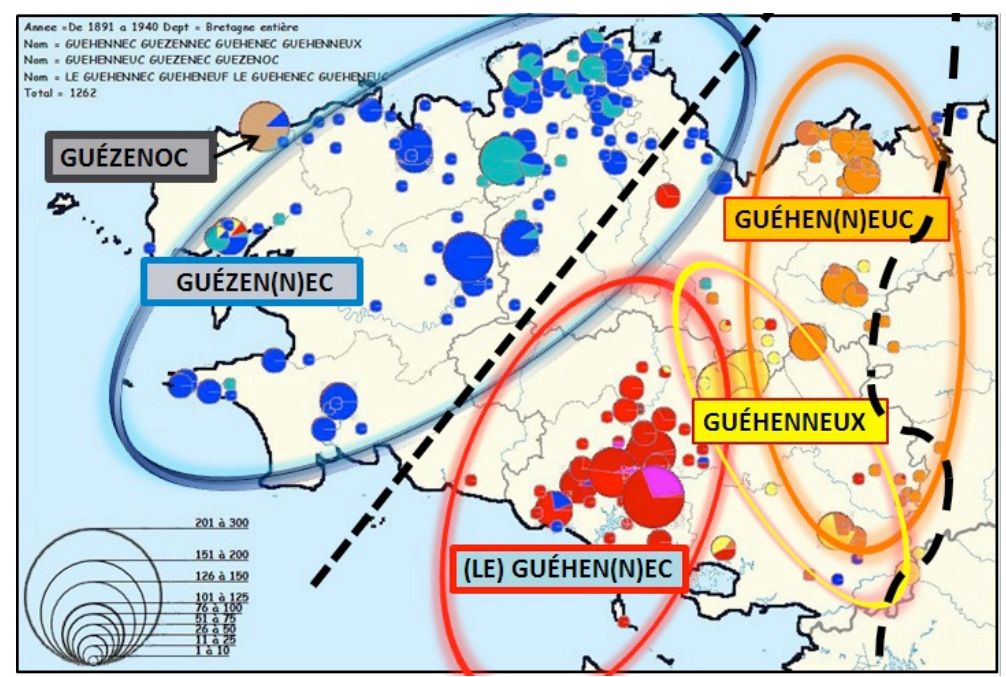

Carte 7. NF apparentés à Guethenoc

Apparenté au vieux-gallois gueithenauc, il est composé à partir du radical vieux-breton uuethen, guethen «combat, bataille» 
(Jackson 1967, 518-520). Les patronymes issus de Guethenoc produisent un nouvel exemple de l'évolution de la fricative $[\Theta]$ du vieux-breton.

Au nord-ouest, le patronyme Guézenoc est localisé dans une seule commune du Finistère septentrional. Nous reviendrons plus loin sur ce suffixe -oc. Le NF Guézen(n)ec révèle lui aussi cette évolution du $[\Theta]$ en $[\mathrm{z}]$. Lorsque nous basculons dans le sud-est, nous constatons à nouveau une prédominance très nette de formes où la fricative interdentale évolue en fricative glottale. Guézen(n)ec devient ainsi (Le) Guéhen(n)ec. Plus à l'est, on relève les NF Guéhenneuc et Guéhenneux dont le suffixe concorde avec les données toponymiques de la partie orientale de la Bretagne (Le Moing 1988).

Une nouvelle fois nous constatons que la répartition des patronymes souligne la bipartition dialectale de l'espace linguistique breton et l'ancienne limite orientale des parlers celtiques aux $\mathrm{XII}^{\mathrm{e}}$-XIII ${ }^{\mathrm{e}}$ siècles. Les celtisants présentent habituellement les noms propres et les noms de lieux en -euc comme l'attestation d'un état de langue ancien remontant au vieux-breton. $\mathrm{Ne}$ faudrait-il pas considérer ses formes relevées en Haute-Bretagne comme les survivances de certains traits phonétiques des parlers celtiques continentaux d'Armorique orientale au haut MoyenAge?

\section{Patronymes en -oc et aire dialectale des suffixes en -og}

L'observation de la variation phonique dans le suffixe de certains patronymes permet de révéler de nouvelles concordances géolinguistiques et anthroponymiques. Prenons le cas du suffixe $-e g$. En général, un syntagme doté de cette terminaison acquiert une valeur d'adjectif. Par exemple, au mot breton beg « pointe » correspond l'adjectif begeg " pointu, avancé ».

Le syntagme peut aussi prendre une valeur de substantif. Il désigne alors le terrain où prolifère le végétal nommé par ce syntagme. On peut citer le terme balan " genêt» et son dérivé balaneg «lieu où pousse du genêt », mesper «nèfles » et mespereg « endroit à nèfles », irin « prunelles » et irineg « terrain à prunelles », tali « laminaires (longues algues brunes)» et talieg « lieu où poussent ces laminaires ». 


\section{LE BRIS}

Dans le nord-ouest de l'espace linguistique breton, l'initiale $[\varepsilon]$ du suffixe -eg s'arrondit et se prononce [o] ou [o]. Ainsi, nous relevons beg-OG au lieu de begeg, balan-OG / balaneg, mesper-OG / mespereg, irin-OG / irineg, tali-OG / talieg, arne$O G$ / arneeg et arnew-OG / arneweg " orageux ».

Relevons également tousOG/touseg «crapaud» et amezOG / amezeg "voisin", tiOG / tieg " paysan, fermier », boutOG / bouteg «panier ». Ces appellations n'appartiennent pas aux catégories précédemment énoncées, mais elles attestent de la même variation vocalique. D'après Ernault et Jackson (Ernault 1905 : 116-118 et Jackson 1967 : 138), tousog s'explique à partir d'un ancien touseg qui aurait lui-même adopté par analogie le modèle du suffixe $-O C$ propre au Léon. Touseg serait luimême un emprunt au roman « tossec » dérivé du latin toxicum.

Passons à présent à l'examen des patronymes. Le logiciel des patronymes nous a permis de dresser la liste des NF dotés du suffice -oc. Il s'agit à l'origine de sobriquets réalisés à partir de substantifs auxquels on a ajouté le suffixe $-o g$ orthographié $-\mathrm{oc}^{4}$. Nous avons ensuite retenus les $15 \mathrm{NF}$ en -oc les plus portés dans l'ensemble des cinq départements afin de les cartographier : Bégoc, Bouzeloc, Bozoc, Croguennoc, Férelloc, Guennoc, Guélennoc, Guézenoc, Louédoc, Perchoc, Quioc, Taloc, Godoc, Lanvoc, Géleoc. Le résultat figure sur la carte 8.

Concernant la période 1891-1940, les NF en -oc sont concentrés pour l'essentiel et de manière assez compacte dans les deux tiers ouest de l'ancien évêché du Léon et de manière apparemment plus importante sur la bande littorale de ce territoire.

Comparons ce résultat à la carte 9 où figurent les naissances de ces mêmes quinze patronymes enregistrées entre 1701 et 1793. Des migrations de commune à commune peuvent manifestement s'être produites sur une distance qui demeure somme toute très réduite.

4 En breton, les consonnes sonores deviennent sourdes en finale absolue ou devant une consonne sourde. Le [g] en finale sera donc prononcé $[\mathrm{k}]$. La consonne sourde a traditionnellement été retenue pour noter la finale de ces patronymes. Cela explique par exemple pourquoi nous avons aujourd'hui un écart orthographique en consonne finale entre l'adjectif breton begog « pointu » et le patronyme Bégoc. 


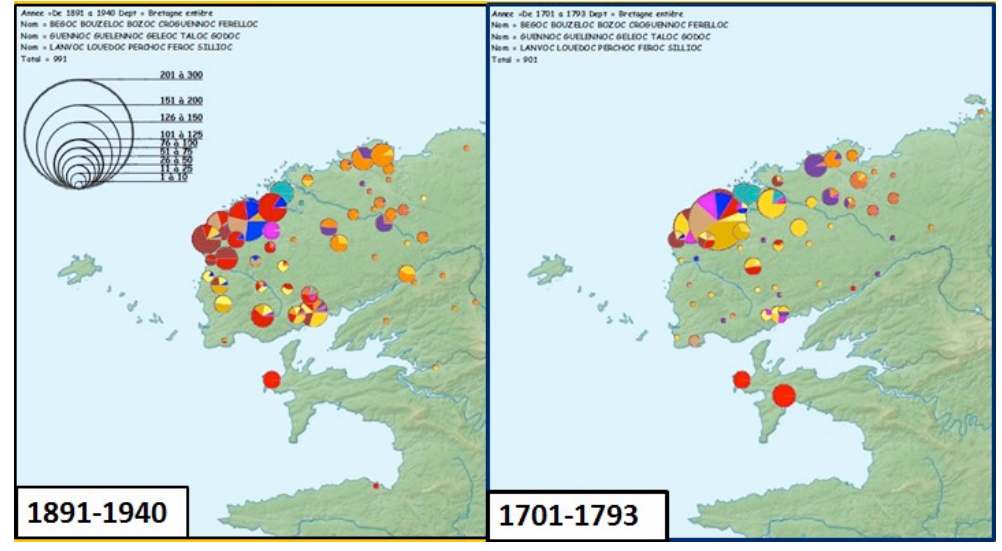

Carte 8.

Carte 9.

Mais, statistiquement la représentation cartographique nous informe surtout que l'ensemble des porteurs de NF en -oc se maintiennent dans la même zone géographique à plus de deux siècles d'intervalle. L'inertie des porteurs des patronymes en -oc est donc frappante à la lumière des cartes.

A titre de comparaison, consultons à présent les cartes du NALBB et de l'ALFMB où il est possible de relever cette variante phonique. Etudions tout d'abord la carte 10 « crapaud » (NALBB 229).

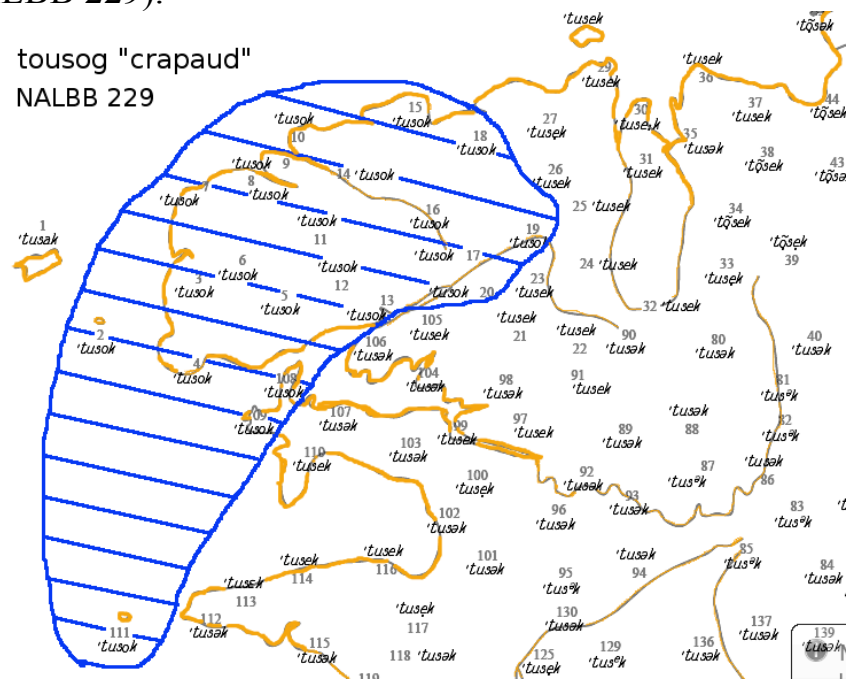

Carte 10. Aire dialectale de tousog «crapaud》 


\section{LE BRIS}

Sur ce document, on constate que l'aire dialectale de tousog s'étend sur les 3/4 ouest du Nord-Finistère; elle inclut aussi l'extrémité de la presqu'île de Crozon et englobe les îles de Molène et de Sein.

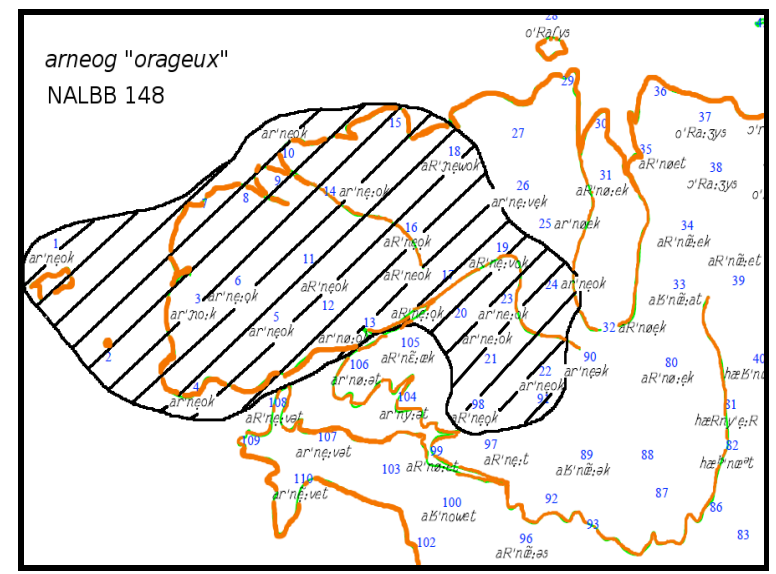

Carte 11. Aire dialectale d'arneog

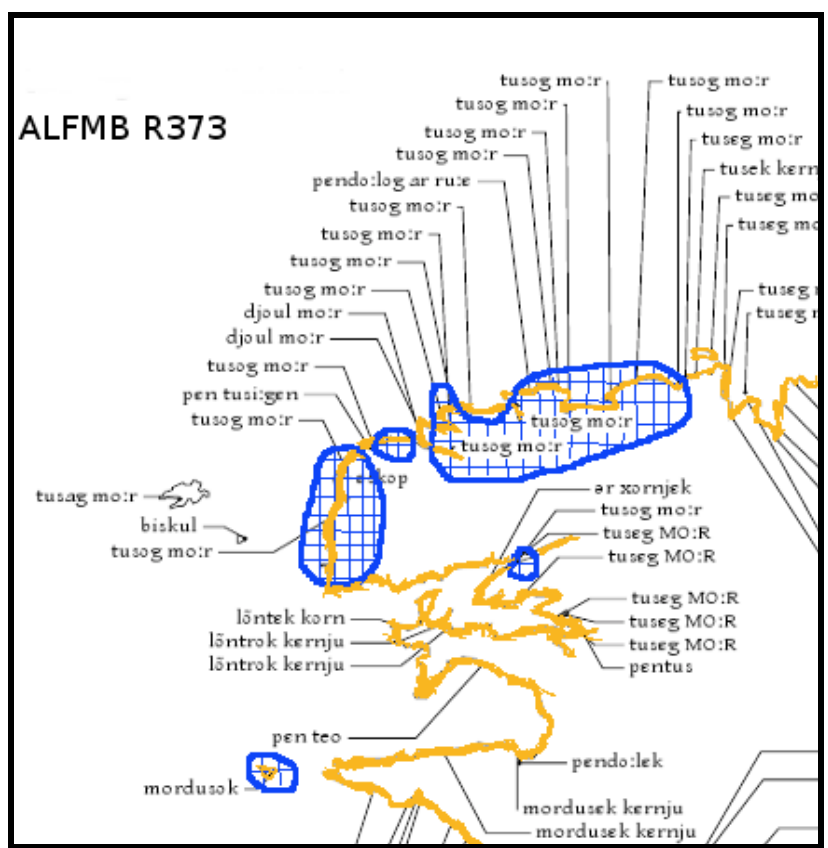

Carte 12. Aire dialectale de tousog-mor " chabot " 


\section{Concordances géolinguistiques et anthroponymiques}

Sur la carte 11 (NALBB 148), l'aire Arneog «orageux » occupe globalement le même territoire que l'aire tousog et s'étend davantage au sud-est vers la zone centrale de BasseBretagne. La presqu'île de Crozon et l'île de Sein sont exclues de cet espace.

La carte 12 (ALFMB R373) représente l'étendue de l'aire correspondant à l'usage de tousog-mor «chabot», lat. cottus gobio. La zone s'étend de manière presque continue depuis l'ouest de l'Île de Batz jusqu'à la Pointe Saint-Mathieu en incluant à nouveau l'Île de Sein.

Le document 13 présente une synthèse des quatre cartes précédemment commentées. Elle permet de préciser la localisation des patronymes en -oc en fonction de la variante phonique $[\varepsilon] /[0]$ ou [o]. La distribution des NF en -oc concorde de manière évidente avec l'aire linguistique où ce suffixe est toujours en usage parmi les parlers bretons actuels.

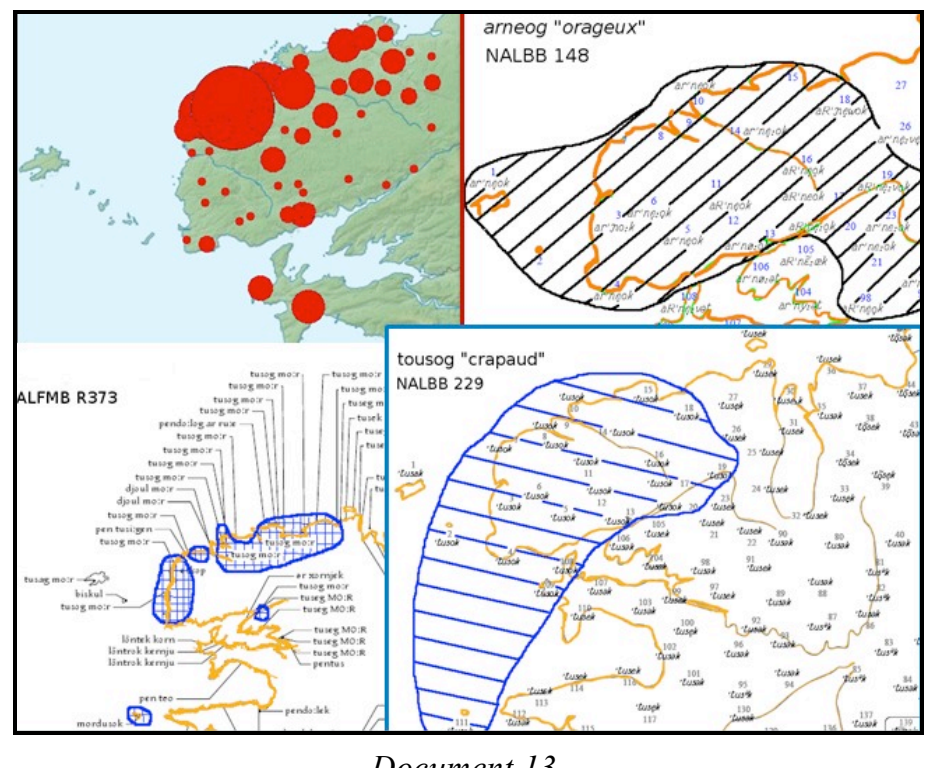

\section{Patronymes en -eur et aire dialectale des suffixes en -eur,} -eureuz

En breton, le suffixe -er permet de constituer un nom d'agent masculin à partir d'un radical verbal. Dans le cas du verbe mag- 


\section{LE BRIS}

$a \tilde{n}$ ['ma:ga] «nourrir », l'adjonction de -er à l'élément de base mag- permet de former mag-er [ma:ger] " (père) nourricier» masc. et mag-erez [ma'ge:rəz/s] «nourrice» fém. Le suffixe féminin -erez est aussi utilisé pour nommer certaines machines, ainsi : dorn-añ « battre (des céréales) », dorn-erez « batteuse ».

Dans le nord-ouest du Finistère, l'initiale vocalique du suffixe est plus généralement prononcée $[\varnothing]$ au lieu de $[\varepsilon]$; la seconde voyelle devient également [ø] par assimilation. Les formes mager et magerez sont ainsi remplacées par les variantes mag-eur et mag-eureuz.

Grâce au logiciel cartographique des patronymes, nous avons considéré les quinze NF les plus fréquents de la période 1891-1940 correspondant à cette variation phonique : Broudeur "personne conduisant le bétail avec un aiguillon », Charreteur " charretier », Corolleur " danseur », Guiadeur «tisserand », Joncqueur " grand-oncle », Lansonneur « le musicien », Larçonneur «le musicien » ${ }^{5}$, Larreur « le laboureur », Larsonneur « le musicien », Magueur "père nourricier», Meneur «carrier», Pastezeur «fabricant de pâté », Podeur " potier », Quemeneur «tailleur », Quidelleur «fabricant ou vendeur de filets de pêcherie ». Ils figurent sur la carte 14 .

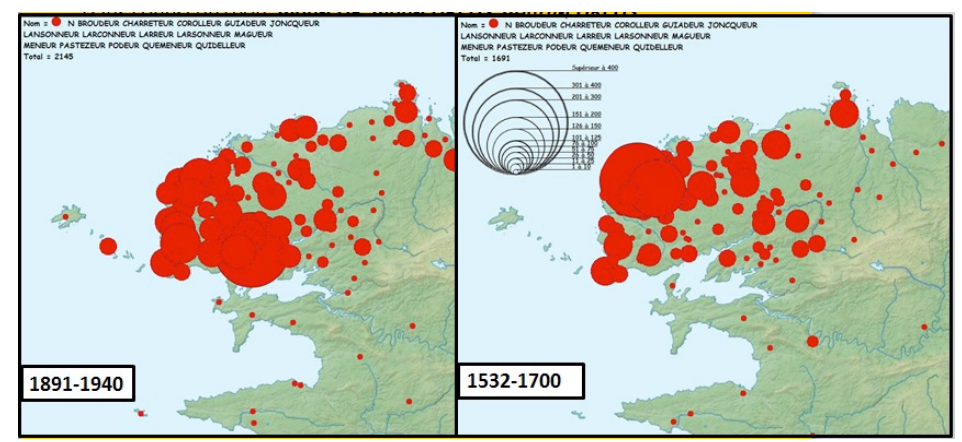

Carte 14

Carte 15.

La carte 14 parle d'elle-même. Semblablement aux NF en -oc, les patronymes en -eur se trouvent à nouveau concentrés dans

${ }^{5}$ Deux articles définis se trouvent agglutinés dans ces deux patronymes : l'article français «l' » et l'article breton an dans le cas de Lan-sonneur; « l'» et ar dans Lar-çonneur. 
le Léon et de manière très compacte à l'extrême ouest de l'ancien évêché. Sur la carte 15, à trois ou quatre siècles d'intervalles, les noms en -eur semblaient déjà être localisés dans le nord-ouest de la Basse-Bretagne. Cela confirmerait à nouveau un certain immobilisme de la population.

Etudions à présent la prononciation de ce suffixe à partir des cartes de l'ALBB et du NALBB. Sur la carte 16 «batteuse » (NALBB 304), la variante dialectale [dur'nø:røs] est indéniablement attestée dans la moitié ouest du Léon.

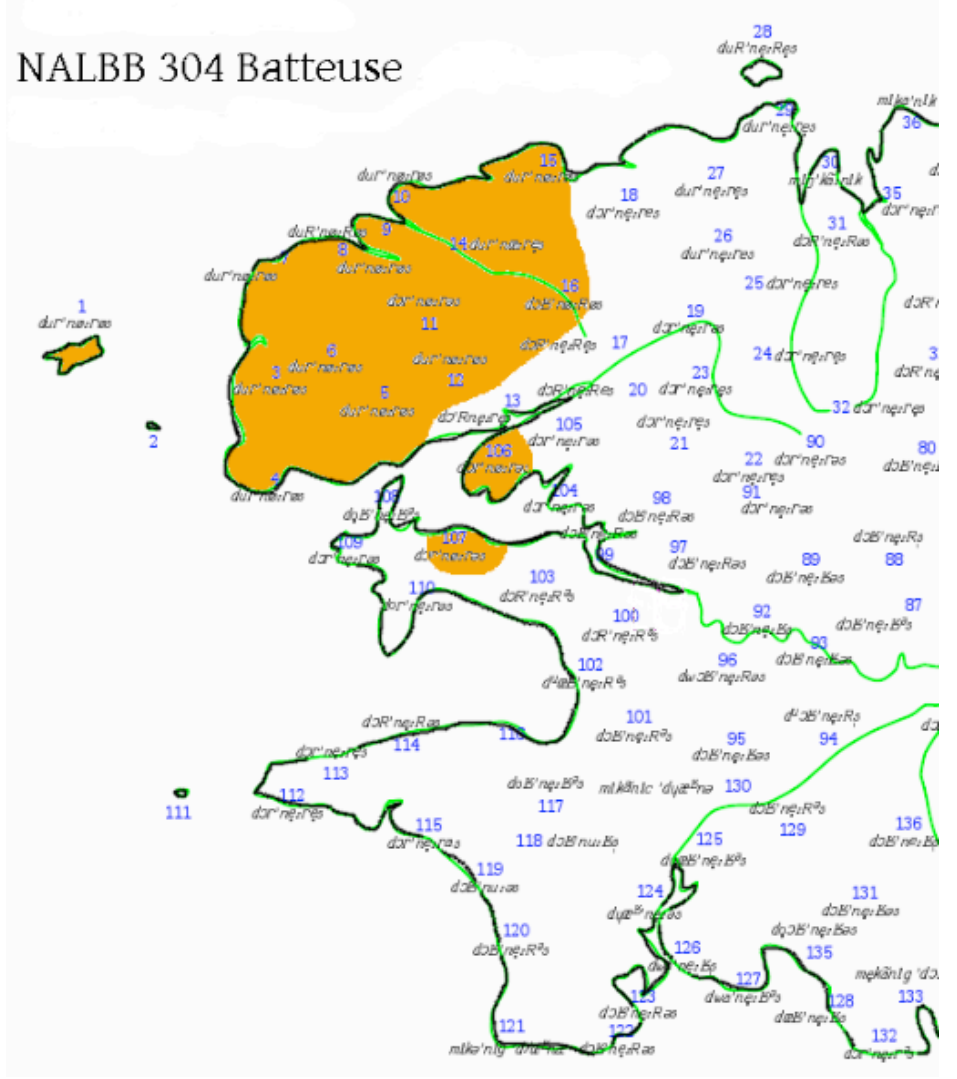

Carte 16. Aire dialectale de dourneureuz «batteuse »

On peut aussi relever ce trait phonétique spécifique du nord-ouest de la Basse-Bretagne sur les cartes NALBB 302 «faucheuse » [fal'hø:røs], NALBB 521 «pêcheur » [pøs'køtør], 


\section{LE BRIS}

ALBB 372 «tailleur» [ke'me:nør], ALBB 387 «boucher» ['ki:gør]. Comme le montre la carte 16, les noms d'agent dotés d'un suffixe contenant une voyelle arrondie sont localisés dans l'aire où la grande majorité des patronymes en -eur a été enregistrée. Une nouvelle fois, on constate une concordance entre les données anthroponymiques et géolinguistiques.

\section{Conclusion}

Cette étude a permis de mettre l'accent sur les concordances entre aires linguistiques et aires culturelles dans la péninsule Armoricaine. Les coïncidences sont parfois frappantes entre la répartition des variantes patronymiques et celle des variantes dialectales encore en usage dans l'espace linguistique breton.

Les fichiers INSEE utilisés pour cette étude concernent l'intervalle historique 1891-1940. Il s'agit donc d'une période relativement récente. Cet intervalle correspond à la période où les enquêtes des atlas linguistiques de Gilliéron et Edmont pour l'Atlas Linguistique de France (Gilliéron \& Edmont 1902-1012) et de Le Roux pour l'ALBB ont été réalisées. Il correspond donc à une France encore très majoritairement rurale et les éléments linguistiques recueillis sont souvent la photographie d'un territoire où les changements et les évolutions se sont produites très lentement, au fil du temps.

Les mouvements de population concernent le plus souvent les classes sociales dirigeantes ou favorisées et semblent toucher assez peu les habitants des zones rurales où se trouvent en majeure partie les locuteurs dialectophones. En utilisant les données du Finistère remontant au XVI ${ }^{\mathrm{e}}$ siècle, on s'aperçoit que la répartition des patronymes sur le territoire est sensiblement identique à celle constatée au début $\mathrm{du} \mathrm{XX}^{\mathrm{e}}$ siècle. Nous ne disposons pas encore de données plus anciennes pour les autres départements bretons, mais si nous parvenions aux mêmes constatations, cela révélerait une relative inertie de la population bretonne sur plusieurs siècles.

Une autre source de documents aurait également pu être examinée : les montres générales et les réformations des fouages de Bretagne dont les plus anciens textes remontent à 1426. Ces recensements servaient à connaître le nombre d'habitants impo- 
sables par évêché afin d'estimer les capacités du duché à supporter un éventuel effort de guerre.

Ces témoignages historiques sont exceptionnels et remarquables. Ils contiennent de nombreuses informations anthroponymiques et toponymiques. Ils peuvent permettre d'étudier assez justement l'origine et la filiation des ancêtres d'une famille précise. Cependant, dans le cadre de cette recherche, leurs données sont difficilement exploitables d'un point de vue statistique. En effet, bien souvent elles ne donnent qu'un simple fragment de la population. Pour certaines paroisses de l'Ancien Régime, on inscrit ainsi les noms des nobles sans relever ceux des personnes de condition sociale plus modeste. Ces documents sont malheureusement trop incomplets pour établir une cartographie des patronymes qui cherche à prendre en compte l'ensemble des individus de la société.

Au travers des exemples que nous avons exposés dans cet article, nous avons vu que la répartition des patronymes peut coïncider de manière assez frappante avec certaines aires linguistiques. Ces correspondances rappellent les structures de base de l'espace linguistique breton telles que Falc'hun les avait déjà énoncées :

- dualité nord-ouest / sud-est des parlers vannetais avec ceux du KLT (KLT : Kerne-Leon-Treger désigne en breton les territoires correspondant aux anciens évêchés de Cornouaille-LéonTrégor) ;

- dualité entre la zone des parlers celtiques et celle des parlers gallo-romans

Elles mettent aussi en évidence des structures de base plus secondaires comme nous avons pu l'observer dans la zone périphérique du nord-ouest.

Jusqu'à quelle époque pouvons-nous faire remonter la structuration du territoire et sa relative immobilité associée à des groupes humains identifiés? Il est difficile de le savoir avec certitude. Nous avons pu observer que la bipartition dialectale nord-ouest / sud-est coïncide avec l'ancienne limite de séparation des territoires gaulois ossisme et vénète. Celle-ci nous ramène à l'Age du Fer, voire du Bronze. Ainsi la structure révélée par la concordance de ces aires culturelles et linguistiques 


\section{LE BRIS}

pourrait s'inscrire dans le paysage depuis l'Age des Métaux dans la façon dont les groupes se partagent les territoires.

Que matérialisent les patronymes en soulignant la valeur plus ou moins marquée de certaines limites? Pour y répondre, il serait par exemple intéressant de connaitre la manière et la période à laquelle ces structures se sont inscrites dans le territoire. Il serait par ailleurs éclairant d'interpréter le rattachement de certains patronymes à ces territoires.

\section{Références bibliographiques}

Brun-Trigaud G., Le Berre Y. \& Le Dû J. (2005). Lectures de l'Atlas linguistique de la France de Gilliéron et Edmont. Paris : CTHS.

Berr A.-G., Le Berre I. \& Le Dû J. (2008). Ichthyonymie bretonne. Atlas Linguistique de la Faune Marine de Bretagne. Brest : Ichthyo.

Darlu P., Degioanni A. \& Ruffié J. (1997). «Quelques statistiques sur la distribution des patronymes en France », Population 3 : 607-634.

Darlu P., Degioanni A., Foulon M. \& Poulain M. (2000). «La distribution des patronymes par rapport à la frontière franco-belge ». Régimes démographiques et territoires : les frontières en question, AIDELF. Paris : PUF, 139-153.

Dauzat A. (1977). Les noms de famille: traité d'anthroponymie française. Paris : Librairie Guénégaud.

Ernault E. (1905). « Notes d'étymologie bretonne », Annales de Bretagne $21: 111-123$.

Falc'hun F. \& Tanguy B. (1966-1970-1979). Les noms de lieux celtiques, 3 vol. Rennes, Bourg-Blanc: Editions Armoricaines.

Falc'hun F. (1981). Perspectives nouvelles sur l'histoire de la langue bretonne. Paris : UGC.

Fleuriot L. (1988). Les origines de la Bretagne. Paris : Payot.

German G. (2007). «Language Shift, Diglossia and Dialectal Variation in Western Brittany : the Case of Southern Cornouaille », in H. L. C Tristram (éd.) The Celtic languages 
in contact: Papers from the workshop within the framework of the XIII International Congress of Celtic Studies. Heidelberg : Universitätsverlag C. Winter, 146-192.

Gilliéron J. \& Edmont E. (1902-12): Atlas Linguistique de France. Paris : Champion.

Gourvil F. (1970). Noms de famille bretons d'origine toponymique. Quimper : Société Archéologique du Finistère.

Humphreys H. L. (1995). Phonologie et morphosyntaxe du parler breton de Bothoa. Brest : Emgleo Breiz.

INSEE (Institut National de la Statistique et des Etudes Economiques) (1999). Fichiers des noms patronymiques de 1891 à 1990 - France entière. Paris : Centre Quetelet-CNRS.

Jackson K. (1953). Language and History in Early Britain. Dublin : DIAS.

Jackson K. (1967). A Historical Phonology of Breton. Dublin : DIAS.

Le Berre A. (2008). Atlas linguistique de la faune marine de Bretagne (ALFMB). Brest : CRBC.

Le Bris D. (2012). « Surnames and geolinguistics in Brittany : a study of concordances ", STUF Language Typology and Universals 65(1) : 95-109.

Le Dû J. (1987). "Un nouveau regard sur l'anthroponymie bretonne », Etudes sur la Bretagne et les pays celtiques, Mélanges offerts à Yves Le Gallo. Brest: CRBC-Institut Culturel de Bretagne, 271-287.

Le Dû J. (1988). «Les appellatifs ethniques dans l'anthroponymie de la Basse-Bretagne ", Campagnes et Littoraux d'Europe, Mélanges offerts à Pierre Flatrès, Hommes et Terres du Nord, $N^{\circ}$ hors série. Lille: Société de géographie de Lille, 67-76.

Le Dû J. (1989). « Anthroponymie et dialectologie : répartition géographique des noms du tisserand d'après les données de l'ALBB, de l'ALF et du fichier INSEE des noms de personnes ", Espaces Romans, Etudes de dialectologie et de géolinguistique offertes à Gaston Tuaillon, vol. II. Grenoble : Ellug, Université Stendhal, 545-559. 


\section{LE BRIS}

Le Dû J. \& Le Berre Y. (1997). "Ce que nomme "breton" », in A. Tabouret-Keller (éd.), Le nom des langues I. Les enjeux de la nomination des langues. Louvain-La-Neuve : Peeters (BCILL), 99-116.

Le Dû J. (2001). Nouvel Atlas Linguistique de Basse-Bretagne (NALBB). Brest : CRBC-UBO.

Le Moing J.-Y. (1988). Toponymie bretonne de Haute-Bretagne : analyse informatique des formes anciennes et modernes. Thèse de $3^{\mathrm{e}}$ cycle. Rennes : Université Rennes 2.

Le Roux P. (1924-63). Atlas Linguistique de Basse-Bretagne $(A L B B)$. Rennes : Plihon et Hommay, Paris : Champion et Droz.

Merlat P. (1959). «Les Vénètes d'Armorique : problèmes d'histoire et d'administration », Mémoires de la Société d'Histoire et d'Archéologie de Bretagne 59 : 5-40.

Mulon M. (2002). Origine et histoire des noms de famille en France. Paris : Errance.

Pitz M. (éd.). Nouvelle Revue d'Onomastique. Paris : Société Française d'Onomastique.

Plonéis J.-M. (1996). L'identité bretonne, l'origine des noms de personnes. Paris : éditions du Félin.

Plourin J.-Y. (1982). Description phonologique et morphologique comparée des parlers bretons de Langonnet et Saint-Servais. Thèse de doctorat d'Etat. Rennes : Université Rennes 2.

Plourin J.-Y. (2010). Les noms de famille et leur histoire. De Quimperlé au port de Pont-Aven. Brest : Emgleo Breiz.

Tanguy B. (1973). Recherches autour de la limite des noms gallo-romains en -ac en Haute-Bretagne. Thèse de $3^{\mathrm{e}}$ cycle. Brest : Université de Bretagne Occidentale.

Tanguy B. (1994). « De l'origine des évêchés bretons », Britannia Monastica 3 : 5-33. 